\title{
Beurteilung der Weiterbildung der Assistenz- ärzte in der Schweiz und in Deutschland (HELIOS Kliniken)
}

\author{
Der FMH-Fragebogen zur Beurteilung der Weiterbildung durch die Assistenz- \\ ärzte wurde von den HELIOS Kliniken in Deutschland übernommen und an \\ ihre Bedürfnisse angepasst. Die Ergebnisse zeigen, dass die Weiterbildung \\ in der Schweiz deutlich positiver beurteilt wird als die Weiterbildung in den \\ HELIOS Kliniken. Die Rücklaufquote bei den HELIOS Kliniken war aber mit 36\% \\ relativ tief.
}

Parwis Fotuhi ${ }^{a}$,

Michael Siegrist ${ }^{b}$,

Pascale Orlow ${ }^{c}$,

Sandra Vogel ${ }^{d}$,

Max Giger ${ }^{e}$

a PD Dr. med., HELIOS Akademie Fulda

b PD Dr., Sozialforschungsstelle der Universität Zürich

c Sozialforschungsstelle der Universität Zürich

d HELIOS Akademie Fulda

e Dr. med., Ressort Medical Education FMH
Korrespondenz:

Dr. med. Max Giger Rosenrain 9

CH-8400 Winterthur

mgiger@hin.ch
In der Schweiz werden die Assistenzärzte jedes Jahr zur Weiterbildung und zur Arbeitssituation befragt [1-3]. Diese jährliche Umfrage, die ein Element der Qualitätssicherung im Bereich der Weiterbildung darstellt, stösst in Deutschland auf grosses Interesse. Die HELIOS Kliniken $\mathrm{GmbH}$, eines der führenden Klinikunternehmen in Deutschland, benutzte den Assistentenfragebogen 2005 der FMH, um die Zufriedenheit mit der Weiterbildung in ihren Kliniken zu erheben.

Viele in der Schweiz praktizierende Ärzte stammen aus dem Ausland. Im Jahre 2005 hatten $37 \%$ der Assistenzärzte in der Schweiz das Staatsexamen im Ausland abgeschlossen. Ein grosser Teil dieser Assistenzärzte kam aus Deutschland. Die Beurteilung der Weiterbildung darf deshalb nicht auf ein Land begrenzt bleiben. Ländervergleiche können aufzeigen, ob neben unterschiedlichen Verdienstmöglichkeiten auch Qualitätsunterschiede in der Weiterbildung eine Rolle spielen könnten, dass die Weiterbildung in der Schweiz absolviert wird.

In der vorliegenden Arbeit werden die Schweizer Daten der Erhebung 2005 mit den Daten von einer Befragung in den HELIOS Kliniken verglichen. Zur HELIOS Gruppe gehören 51 Kliniken mit insgesamt 15200 Betten. Von den rund 2700 Ärzten, die im Konzern angestellt sind, befinden sich ungefähr 1100 in Weiterbildung. Die Weiterbildung der Assistenzärzte in Deutschland weist strukturelle Ähnlichkeiten mit der Weiterbildung in der Schweiz auf. Die Assistenzärzte arbeiten mehrere Jahre in einem Krankenhaus oder einer Arztpraxis. Ziel der Weiterbildung ist die Erlangung eines Facharzttitels auf einem bestimmten Gebiet.

\section{Evaluation de la formation post-} graduée des médecins-assistants en Suisse et en Allemagne (cliniques HELIOS)

Le questionnaire de la FMH pour l'évaluation de la formation postgraduée par les médecins-assistants a été repris en Allemagne par les cliniques HELIOS qui l'ont adapté à leurs besoins. Les résultats montrent que la formation postgraduée est évaluée de manière nettement plus positive en Suisse que dans les cliniques HELIOS. Le taux de réponses de $36 \%$ pour les cliniques HELIOS était toutefois relativement bas.

\section{Methode}

\section{Stichprobe}

Die Schweizer Stichprobe wurde bereits in einer anderen Publikation in der SÄZ detailliert beschrieben [3]. Die Befragung wurde zwischen Juli und November 2005 durchgeführt, und die Rücklaufquote betrug $68 \%$.

Die Fragebogen wurden den Assistenzärzten von HELIOS an ihre Privatadresse geschickt. Die ausgefüllten Fragebogen konnten direkt an die HELIOS Akademie in Fulda zurückgeschickt werden. Insgesamt wurden 1079 verschickt. Die Rücklaufquote betrug 36\% ( $\mathrm{N}=389) .51 \%$ der Fragebogen wurden von Assistenzärztinnen und 
49\% von Assistenzärzten ausgefüllt. Die Befragung wurde vom 8. März bis 7. April 2006 durchgeführt.

\section{Fragebogen}

Der Schweizer Fragebogen der FMH wurde für die Befragung bei HELIOS zum grossen Teil unverändert übernommen, damit ein Vergleich mit den Schweizer Daten möglich wird. In erster Linie mussten die Befragten zu Aussagen Stellung nehmen. Dabei standen bei den meisten Items sechs Antwortkategorien zur Auswahl, von 1 «trifft überhaupt nicht zu» bis zu 6 «trifft voll und ganz zu».

Aufgrund von theoretischen und statistischen Überlegungen wurden die Aussagen zu Skalen zusammengefasst, die im folgenden beschrieben werden. Für die Abschätzung der Skalengüte wurde Cronbachs Alpha verwendet. Dieser Koeffizient kann Werte zwischen 0 und 1 annehmen. Je höher der Wert, desto grösser ist die interne Konsistenz einer Skala. Die Reliabilitätskoeffizienten für die Schweizer Befragung 2005 und die Befragung bei HELIOS sowie beispielhafte Fragen sind in Tabelle 1 aufgeführt. Die meisten Skalen weisen in beiden Ländern sehr gute Werte für die interne Konsistenz auf. Einzig die Skala Fehlerkultur hat in beiden Stichproben einen tiefen Wert.

\section{Ergebnisse}

\section{Beurteilung der Weiterbildung}

Die Durchschnittswerte der acht Dimensionen für die Schweiz und für HELIOS sind in Tabelle 2 aufgeführt. Bei allen Dimensionen konnte in der Schweiz ein signifikant höherer Wert als bei den HELIOS Kliniken beobachtet werden. Der grösste Unterschied konnte für Evidence-based Medicine beobachtet werden. Obwohl hier auch in der Schweiz die Werte relativ tief ausfallen, war der Wert für HELIOS noch deutlich tiefer. Der geringste Unterschied ergab sich für die Dimension Fachkompetenz.

\section{Theoretische Weiterbildung und Arbeitszeit}

In der Schweiz ist das Angebot für theoretische Weiterbildung grösser als bei den HELIOS Kliniken. In der Schweiz beträgt der Median 3 Stunden für die angebotene theoretische Weiterbildung, bei den HELIOS Kliniken beträgt der Median 1 Stunde pro Woche. In der Schweiz (Median = 2 Stunden) nutzen die Assistenzärzte die theoretische Weiterbildung häufiger als die Assistenzärzte der HELIOS Kliniken (Median = 1 Stunde).

Der Median der wöchentlichen Arbeitszeit lag 2005 in der Schweiz bei 52 Stunden. In den HELIOS Kliniken war der Median deutlich höher

Tabelle 1

Die wichtigsten Skalen.

\begin{tabular}{|c|c|c|c|c|}
\hline Skala & Beispiel für Items & Anzahl Items & Alpha Schweiz & Alpha HELIOS \\
\hline Globalbeurteilung & Ich würde die Weiterbildungsstätte weiterempfehlen. & 3 & .93 & .92 \\
\hline Fachkompetenz & $\begin{array}{l}\text { Wie gross ist der Beitrag der Weiterbildungsstätte, an der } \\
\text { Sie zurzeit tätig sind, zur Entwicklung der folgenden } \\
\text { Kompetenzen, Fähigkeiten beziehungsweise Kenntnisse? } \\
\text { A) Differentialdiagnostisches Denken }\end{array}$ & 28 & .96 & .95 \\
\hline Lernkultur & $\begin{array}{l}\text { Meine Weiterbildner nehmen sich genügend Zeit, um mir } \\
\text { Zusammenhänge und Fragen zu erklären, die ich nicht } \\
\text { verstanden habe. }\end{array}$ & 6 & .89 & .89 \\
\hline Führungskultur & $\begin{array}{l}\text { Meine Vorgesetzten sorgen für eine gute Zusammenarbeit } \\
\text { im Team. }\end{array}$ & 6 & .91 & .91 \\
\hline Fehlerkultur & $\begin{array}{l}\text { An unserer Weiterbildungsstätte gibt es ein System zur } \\
\text { Meldung kritischer Ereignisse und Komplikationen oder } \\
\text { ein CIRS (Critical Incidence Reporting System). }\end{array}$ & 3 & .50 & .58 \\
\hline Entscheidungskultur & $\begin{array}{l}\text { Es wird vorgelebt, dass bei einer Entscheidung alle Aspekte } \\
\text { (soziale, psychische und medizinische) berücksichtigt } \\
\text { werden müssen. }\end{array}$ & 2 & .91 & .92 \\
\hline Betriebskultur & $\begin{array}{l}\text { In unserer Weiterbildungsstätte (Ärzte und Pflegepersonal) } \\
\text { herrscht ein gutes Arbeitsklima. }\end{array}$ & 4 & .89 & .85 \\
\hline Evidence-based Medicine & $\begin{array}{l}\text { Ich lerne, die Ergebnisse einer wissenschaftlichen Studie } \\
\text { konkret bei der Behandlung eines Patienten anzuwenden. }\end{array}$ & 4 & .93 & .94 \\
\hline
\end{tabular}

Anmerkung: Schweiz N = 2686-5705; Helios N = 201-389. Die Stichprobengrössen schwanken relativ stark, weil bei der Fachkompetenz nicht alle Befragten alle Fragen beantworten konnten. 
Tabelle 2

Mittelwerte und Konfidenzintervalle für die acht Skalen für die Schweiz und für die HELIOS Kliniken.

\begin{tabular}{llllll} 
& \multicolumn{2}{l}{ Schweiz } & \multicolumn{2}{l}{ HELIOS } \\
\hline Skalen & $\mathrm{M}$ & $95 \% \mathrm{KI}$ & $\mathrm{M}$ & $95 \% \mathrm{KI}$ \\
\hline Globalbeurteilung & 4,84 & $4,82-4,87$ & 3,99 & $3,85-4,13$ \\
\hline Fachkompetenz & 4,67 & $4,65-4,70$ & 4,15 & $4,06-4,24$ \\
\hline Lernkultur & 4,72 & $4,70-4,75$ & 3,87 & $3,76-3,99$ \\
\hline Führungskultur & 4,73 & $4,71-4,76$ & 4,04 & $3,93-4,14$ \\
\hline Fehlerkultur & 4,32 & $4,27-4,36$ & 3,43 & $3,25-3,61$ \\
\hline Entscheidungskultur & 4,87 & $4,84-4,90$ & 4,04 & $3,90-4,18$ \\
\hline Betriebskultur & 4,94 & $4,92-4,97$ & 4,44 & $4,33-4,55$ \\
\hline Evidence-based Medicine & 3,54 & $3,51-3,58$ & 2,28 & $2,15-2,41$
\end{tabular}

Anmerkungen: Schweiz N = 5624; HELIOS N = 384 . lenschlüssel in den Kliniken und eine höhere Bezahlung bis zur differenten Weiterbildungsordnung und Ärztekammerstruktur in Deutschland und der Schweiz.

Weiterhin war die Rücklaufquote der Umfrage in den HELIOS Kliniken mit 36\% massiv tiefer als die Rücklaufquote in der Schweizer Befragung (68\%). Es kann nicht ausgeschlossen werden, dass bei den HELIOS Kliniken stärker die unzufriedenen Assistenzärzte mitgemacht hatten und deshalb die Ergebnisse schlechter ausfielen als in der Schweiz. Weiter muss beachtet werden, dass in der Schweiz alle Weiterbildungsstätten an der Umfrage teilnehmen. Auch wenn die HELIOS Kliniken wahrscheinlich exemplarisch für die Weiterbildungssituation in Deutschland sind an der Umfrage beteiligten sich HELIOS Kliniken mit 100 Betten bis zur nichtuniversitären Klinik der Maximalversorgung mit 1300 Betten -, können aufgrund der vorliegenden Ergebnisse noch keine generellen Aussagen über die Zufriedenheit der Assistenzärzte in Deutschland gemacht werden. Es wird interessant sein, die Ergebnisse einer deutschlandweiten Befragung durch z.B. die deutsche Bundesärztekammer mit der Situation in der Schweiz zu vergleichen. Neben einem internen Benchmark erhalten die beteiligten deutschen Kliniken dann auch einen internationalen Benchmark. Eine solche Untersuchung könnte auch in Deutschland ein wichtiges Element der Qualitätssicherung im Bereich der Weiterbildung sein. sionen zu. Auch scheinen die Mitarbeiter der HELIOS Kliniken tendenziell weniger Stunden Weiterbildung zu besuchen als ihre Kollegen in der Schweiz.

Die vorliegende Befragung der Ärzte in Weiterbildung ist der erste direkte Vergleich der Weiterbildungssituation in deutschen Kliniken mit der Situation in der Schweiz. Bei der Interpretation der Ergebnisse ist es wichtig zu bedenken, dass Unterschiede in den Gesundheitsund Weiterbildungssystemen beider Länder bestehen. Diese Unterschiede reichen von unterschiedlichen Arbeitszeitgesetzen und Krankenhausfinanzierung über verschiedenartige Stel-

\section{Literatur}

1 Siegrist M, Gutscher H, Giger M. Was haben Betriebskultur, Motivation und Fremdjahr mit der Weiterbildung zu tun? Schweiz Ärztezeitung. 2004;85(15):783-91.

2 Siegrist M, Orlow P, Giger M. Weiterbildung aus der Sicht der Assistenzärzte: Die wichtigsten Resultate der Umfrage 2004 bei Assistenzärztinnen und -ärzten über die Weiterbildung. Schweiz Ärztezeitung. 2005;86(7):412-23.

3 Siegrist M, Orlow P, Giger M. Weiterbildung und Arbeitssituation aus Sicht der Assistenzärzte. Schweiz Ärztezeitung. 2006;87(10):379-86. 